\title{
Interactive comment on "Refining physical aspects of soil quality and soil health when exploring the effects of soil degradation and climate change on biomass production: an Italian case study" by Antonello Bonfante et al.
}

\section{Rossiter (Referee)}

david.rossiter@wur.nl

Received and published: 24 September 2018

Review of soil-2018-30 D G Rossiter ISRIC-World Soil Information/Cornell University/Nanjing Normal University

(1) General comments

This paper is a welcome step towards quantifying the concept of "soil health" and towards relating it to the concept of soil phenoforms (management-induced semipermanent changes in soil properties within one soil genoform). It also presents a 
convincing argument to use simulation for the future (obviously). The technical aspects are sound, in particular a good choice of soil-plant-atmosphere model and associated SOILD pedotransfer functions and a good choice of quantitative phenoform indicators. Less convincing are the future scenarios, although that is entirely because of the uncertainty in the RCP 8.5- IPCC scenario - a reasonable choice since this is what is presented to policy makers. The clear message is that biomass yield, as affected by changes in soil physical properties, can be a quantitative indicator of soil physical "health".

The paper mentions an "logical and interconnected sequence considering pedological, physical, chemical and biological aspects" to holistically evaluate soil health; however the paper does not give any details of how such a sequence would work, nor indeed why a sequential approach (and in the order given, at that) would be desireable. This is outside the scope of the paper (as indicated by its title) but if it is included in the discussion it could be expanded somewhat.

(2) Specific comments

L30 likely under the scenarios; see also comment below on L309

L57 fixed values as expressed by laboratory measurements of the pressure head

L91 Unfortunately, the "soil series" is not used everywhere, explain that the lowest level of other classifications are essentially the same concept. However this level is recognized as necessary for communication with stakeholders, see for example: Lepsch, I. F. (2013). Status of soil surveys and demand for soil series descriptions in Brazil. Soil Horizons, 54(2), 0. https://doi.org/10.2136/sh2013-54-2-gc

L182: Is Yw always lower than Yp? Perhaps if averaged over a number of years - there are always unfavourable years.

Printer-friendly version

L200 These are the phenoforms! emphasize

L255, Figure 1: terminology "environmental systems" seems over-ambitious for what are "landform classes" or similar. Is this the standard terminology used in Italian soil 
survey?

L259 It isunclear how these decision trees work

SOILD

L309 will -> are expected to (under the scenarios); this is correct in o.a. L329 "Projected effects..."

Interactive

L354 also in the USA soil series names are often used in advertising for farmland, as

comment well as by agricultural consultants

L362, conclusions: Suggest to use the full term "soil [genoforms, phenoforms]" throughout, for consistency with Rossiter \& Bouma (2018), cited in the paper.

L373 More detail on what would be a "logical and interconnected sequence considering pedological, physical, chemical and biological aspects", or leave this outside of the scope of this paper.

L385-7 Conclusion point 3. Can these models also cover the biological aspects as proposed in the paper?

L391-2 Conclusion point 5. Not established in this paper.

Figure 2 shows "error bars" for Yw simulations, but neither the text nor the figure caption explain how these are derived. Is this from simulating each year (the uncertainty) and then averaging (the bar)? Similarly for Figure 3, why do we have a boxplot and not just one value per reference? \S2.3 (Simulation modelling) does not make it clear that the simulations were run per year (I think) for each 30-year period. Neither is this clear from $\backslash S 2.5$ (Climate information); L280 only mentions the full period, and does not even break it down into the 30-year intervals that are shown in the Figures and in $\backslash$ S3.1.

Figure 2 caption "over all soil series" is in fact only taking into account the area's climate and an ideal profile, so it has nothing to do with the series.

(3) Technical corrections 
Please run a spelling checker

Awkward use of - in abstract

SOILD

Please be consistent: either Fig. or Figure in the text.

L41 discourse

Interactive

L55 penetrometer

comment

L62 quote " not"

L69 ) $\rightarrow>-$

L95 (2016)

L102-3 run-on sentence with two different ideas

L119-123 and further in this section: inconsistent use of arabic or small roman numerals, with or without parentheses

L132 add: "in three ways": (1)...

L171 pedotransferfunctions -> pedotransfer functions

L211 etc. scenario's [Dutch] -> scenarios [English]

L232 reference to Soil Taxonomy (which version?)

L257 (Soil, 1999) not correct; also L515 in references

L280 "The simulation was performed cover the period" not grammatical; I think this means "The simulations covered the period..."

L372 Indicators -> indicators

Printer-friendly version

L374 [b]iological

Discussion paper

L376 Carolina 
Figure 5 caption infiltrativce -> infiltration

Table 2 R di Pearson -> Pearson's R

SOILD

Interactive comment on SOIL Discuss., https://doi.org/10.5194/soil-2018-30, 2018.

Interactive

comment

Printer-friendly version

Discussion paper 